\title{
Pureza y erotismo en la poesía de Oliverio Girondo
}

\author{
Guillermo LESCANO ALLENDE \\ Universidad Nacional Autónoma de México
}

Los nervios se me adhieren al barro, a las paredes, abrazan los ramajes, penetran en la tierra, se esparcen por el aire, hasta alcanzar el cielo.

Oliverio Girondo, "Comunión plenaria"

En sus reflexiones de 1936 dedicadas a la pintura moderna, Oliverio Girondo se muestra consciente de la quiebra del arte con las viejas normas de representación de la realidad. Así, caracteriza al cubismo como una corriente para la cual la creación artística se transforma en un acto mental:

Dentro de los límites de su tela, el artista se halla en un trance semejante al de Dios ante la Nada. Su creación ha de surgir de su propia sustancia y ha de someterse a las leyes que le impone su voluntad omnipotente. Cuando apela a lo exterior, cuando incurre en la debilidad de manifestar alguna ternura por un objeto humilde (vaso, periódico, botella) le inflige las deformaciones que se le antojan, lo somete a la estructura y al ritmo que requiere la composición.'

I "Pintura Moderna". (Colección de Rafael A. Crespo.) Prólogo de Oliverio Girondo. (Exposición en el Museo Nacional de Bellas Artes, Buenos Aires, 14 de agosto al 6 de septiembre de 1936.) Reproducido en Obras completas, p. 218 . En lo sucesivo, todas las citas de Girondo provienen de esta edición.

Las Obras completas de Oliverio Girondo permanecieron agotadas hasta 1990, año en que se reeditaron con el titulo Obras, con un total de cuatrocientas sesenta páginas, esto es, con veintisiete páginas menos. La diferencia numérica estriba en que la segunda edición no incluye la bibliografia de Horacio Jorge Becco. Se titula Obras debido a que en 1987 Jorge Schwartz recopiló una serie de textos inéditos de Girondo que incluyó, con otros documentos vinculados al autor y una bibliografia más actualizada que la de Becco, con el nombre Homenaje a Girondo (Corregidor, Buenos Aires, 1987). 
De este modo se hace evidente que para Girondo, como para varios representantes de las vanguardias, el problema de la relación arte/representación ocupaba un lugar central.

Oliverio Girondo (1891-1967), poeta argentino, viajero incansable y creador estrechamente ligado a las vanguardias, dio al mundo una obra singular y definió una peculiar trayectoria creativa que se puede caracterizar, parafraseando un poema suyo, como un "vuelo sin orillas". Esta particular y muy productiva carrera (iniciada en 1922 con la publicación, en París, de Veinte poemas para ser leidos en el tranvía) quedó, como la de tantos otros escritores argentinos de su generación, "sepultada" por la emergencia de la figura de Borges y sólo recientemente, y de manera muy parcial, ha sido revalorizada.

\section{Oliverio Girondo ante la crítica}

Existe, por cierto, una copiosa bibliografia constituida por estudios críticos, ensayos, reseñas y artículos periodísticos sobre Girondo, escritos desde 1922, que Horacio Jorge Becco recopiló para incluirla en la edición de sus Obras completas (1968). Los trabajos más brillantes siguen siendo, sin duda, los de los poetas Aldo Pellegrini y Enrique Molina. Desde 1968 en adelante Girondo es objeto de estudio por parte de académicos y universitarios de diversos países.

En la década de los setentas hubo un primer descubrimiento crítico de la obra de Girondo por parte de estudiosos argentinos como Beatriz de Nóbile y Gaspar Pío del Corro. Pero fueron los agudos estudios de Jorge Schwartz los que contribuyeron a repensar y a releer debidamente la obra de Girondo.

Existe además una serie de tesis de grado dedicadas al poeta, en su mayoría inéditas. ${ }^{2}$ De ese corpus comento las siguientes, en orden cronológico.

En primer lugar, Beatriz de Nóbile ${ }^{3}$ ubica a Girondo en el contexto de la literatura cubista, con influencias de Guillaume Apollinaire y antecedentes inmediatos en Paul Morand y Blaise Cendrars. Con relación a Morand, en

${ }^{2}$ Cito las tesis doctorales de Mihai Grunfeld, Oliverio Girondo y la poesia de viaje; Daniel E. Nelson, Five central figures in Argentine avant-garde art and literature: Emilio Pettoruti, Xul Solar, Oliverio Girondo, Jorge Luis Borges, Norah Borges; Olga Juzyn-Amestoy, Oliverio Girondo: la realidad de la palabra. Hay en México una tesis de licenciatura inédita escrita por Patricia Gola: Vanguardismo y ruptura en la poesia de Oliverio Girondo.

${ }^{3}$ Beatriz de NóBILE, El acto experimental. Oliverio Girondo y las tensiones del lenguaje. 
nuestra opinión, es difícil probar la deuda de Girondo, ya que el poeta francés comienza a publicar en 1922 ( $\mathrm{y}$ la obra posterior del poeta argentino se aleja definitivamente de esta presunta influencia). No obstante, Morand y Girondo comparten el "esprit nouveau" (denominación que Ortega y Gasset traduce como "nueva sensibilidad"), que propone, entre otras innovaciones y rupturas, el fragmentarismo y la simultaneidad. Agreguemos que el poema de Morand, "L'heure vraie ou Brahma voit le monde en une fois", 4 es significativo desde el mismo título: la mirada ubicua del poeta aprehende simultáneamente el mundo. El texto aprovecha en forma poética los términos característicos del cubismo: "je mets sur un rang les pays, les races, / et je les mitraille toutes avec un seul coup d'oeil, / une seule pensée".

La relación con Cendrars se basa en la poesía de viajes; pero éste es intimista: sus poemas se identifican con un "yo lírico", de filiación romántica. Girondo, según De Nóbile, sigue las técnicas cubistas: cosmopolitismo, preocupación por la simultaneidad, fragmentación de la realidad. Y añade a estos elementos la carga de humor, erotismo y antisolemnidad. La hipérbole distorsiona los componentes mencionados, por lo que habría, en Girondo, la tendencia a degradar lo que describe.

El trabajo de Beatriz de Nóbile tiene el gran mérito de ser el primer estudio de carácter académico dedicado a nuestro autor. La amistad que esta crítica mantuvo con Norah Lange (escritora destacada, mujer autónoma y audaz, que llegó a ser considerada la "Musa de Florida" y, desde 1943, esposa de Girondo) le permitió, además, tener el acceso a fuentes directas (que han servido en grado sumo, justo es reconocerlo, a otros investigadores). Su tesis constituye, en mi opinión, una interesante disección estructural de la obra girondiana.

Gaspar Pío del Corro 5 analiza la poesía de Girondo desde la óptica del grotesco como una forma de deformación de la realidad descrita. Destaca los valores negativos que Girondo exalta en personas y cosas. Para Del Corro la poesía de Girondo es expresionista por el hecho de que hay en ella una voluntad distorsionadora de la realidad según las demandas del yo subjetivo. La poesía de Girondo propone, en opinión de este crítico, una inversión de los valores. El profesor Pío del Corro maneja un vasto caudal bibliográfico y posee una sólida formación semiológica. Pero hay en su trabajo un exceso de juicios de valor precisamente porque juzga, desde la ética, la presencia de elementos, en su opinión, negativos. Cito una frase referida a Veinte poemas para ser leidos en el tranvía: "entre las apariencias positivas de los

\footnotetext{
${ }^{4}$ Paul MORAND, Papiers d'identité.

${ }^{3}$ Gaspar Pío del CORRo, Oliverio Girondo. Los limites del signo.
} 
signos de vitalidad, asoman rastros inconfundibles de valores negativos o antivalores significados por la forma de adjetivación" (p. 29).

Jorge Schwartz, ${ }^{6}$ especialista en las vanguardias, lleva a cabo el estudio más exhaustivo dedicado al poeta; gran parte de su tesis indaga en las vanguardias latinoamericanas. Según Schwartz, la poesía de Girondo de los años veintes se caracteriza por el uso de la prosa y el verso libre. Indica, como elementos comunes entre Girondo y Oswald de Andrade, la enumeración, recurso procedente de Whitman. Destaca la influencia de la muchedumbre y del flaneur en la poesía urbana. El cubismo, y el futurismo en particular, impusieron la "modernolatría" y la "maquinolatría". La poesía busca la ubicuidad por medio del dislocamiento geográfico. El cine y la fotografia irrumpen en los montajes poéticos. El desarrollo del turismo propició las formas cinéticas de la poesía. En fin, propone Schwartz, en el poeta argentino subyace una visión carnavalesca por su voluntad de mezclar los géneros.

Sostengo que Girondo está muy lejos de la admiración por la máquina, rasgo sí atribuible, por ejemplo, a Marinetti. El símbolo girondiano de modernidad y urbanidad es el modesto tranvía; también lo es el vapor, símbolo de una modernidad y un cosmopolitismo controlados. Pero difiere de Marinetti en la actitud ante otras máquinas: el poeta argentino no hace la apología de la máquina y el progreso sino que somete las novedades tecnológicas a un trabajo de estilización y simbolización.

Con posterioridad, Schwartz ha seguido interesado en Girondo. Sobre las relaciones con la poesía concreta, abundó en "Vanguardias enfrentadas: Oliverio Girondo y la poesía concreta". ${ }^{7}$ En 1984 colaboró con el artículo "¿A quién espanta el espantapájaros?", en el número 6 de la revista $X u l^{8}{ }^{8}$ dedicado a Girondo, en el contexto del resurgimiento democrático en Argentina.

El más reciente trabajo de Schwartz data de 1995, "A trajetória maismedular de Oliverio Girondo", 9 donde refrenda sus tesis iniciales y postula otras que, en mi opinión, resultan forzadas. Por ejemplo, sostiene que Girondo es un poeta barroco. Estimo que, al cabo de los años, categorías como "barroco", "barroquización" y "carnavalización", entre otras, se han

"Jorge SChWARTZ, Vanguardia y cosmopolitismo en la década del veinte: Oliverio Girondo y Oswald de Andrade. Esta tesis doctoral fue posteriormente publicada con el mismo título. San Paulo, Perspectiva, 1983.

${ }^{7}$ Maldoror, 16 de noviembre de 1982, pp. 22-35.

${ }^{8}$ Buenos Aires, mayo de 1984 , pp. 30-36.

" Cf. J. SChwartz, "A trajetória maismedular de Oliverio Girondo", en Oliverio Girondo. A pupila do zero, pp. 131-150. 
convertido en comodines - o en modas en el peor de los casos- que estimulan abusos metodológicos con los que se arriba a soluciones fáciles por parte de los críticos. No obstante, en este reciente ensayo hace inteligentes precisiones en torno al uso del heptasílabo en En la masmédula (1956), fenómeno detectado por Aldo Pellegrini y que Schwartz se encarga de desarrollar.

Además de la labor crítica, hay que destacar la importantísima recopilación de documentos (textos inéditos de Oliverio Girondo, entrevistas, correspondencia y actualización bibliográfica) que Jorge Schwartz reunió con el título de Homenaje a Girondo. ${ }^{10}$

Los esfuerzos de renovación crítica de los setentas quedaron en cierto modo interrumpidos por la dictadura militar y la dispersión de muchos estudiosos. Se continuaron con posterioridad a 1983 junto con una nueva valoración de Borges, releído desde entonces por la crítica como nuevo paradigma escritural. No es casual que la reapertura del interés crítico por la idea de posmodernidad coincida con un renovado interés por experiencias atípicas como la de Girondo. El trabajo de Jorge Schwartz, arriba citado, fue realizado en San Paulo, y los dos siguientes en universidades de Estados Unidos.

Mihai Grünfeld, " ya en los ochentas, retoma las observaciones de Schwartz e incursiona en el análisis gramatical de Veinte poemas para ser leídos en el tranvía (1922) y Calcomanias (1925). Indaga en la poesía de Valery Larbaud, como antecedente decisivo de Girondo. Establece semejanzas entre el heterónimo de Larbaud, A. O. Barnabooth, y Girondo. En la invención de Larbaud, Barnabooth es un millonario nacido en Arequipa que viaja por el mundo y describe, en particular, Europa, tal como lo hará Girondo, con un dejo de mundanidad. Resulta estrecha la comparación porque Girondo siempre se escapa del intimismo. Larbaud, como Cendrars y Morand, tiene una base romántica.

Es sorprendente que el trabajo de Grünfeld, cuyo tema es la poesía de viajes, no incluya En la masmédula. Omisión extraña puesto que toda la crítica, bien conocida por él, admite que la última obra de Girondo es la culminación, viaje interior, del recorrido iniciado en 1922.

Olga Juzyn-Amestoy ${ }^{12}$ aprovecha en su tesis los estudios de Schwartz y a partir de ellos incursiona en la poesía de Girondo mediante la aplicación de algunos postulados de Peter Bürger: la vanguardia se propuso devolver el arte a la praxis. Polemiza con Jorge Schwartz al negar que en Girondo exista lo carnavalesco bajtiniano en un espacio abierto y lo plantea como un

${ }^{10}$ Buenos Aires, Corregidor, 1987.

${ }^{11} \mathrm{M}$. GRUNFELD, op. cit.

${ }^{12} \mathrm{O}$. JUZYN-AMESTOY, op. cit. 
espacio cerrado... Pero, en rigor, el trabajo de Juzyn-Amestoy es también una aplicación de Bajtin, con los términos invertidos. La principal limitación de esta tesis es la de un compromiso previo con ciertos presupuestos teóricos que vuelve esquemático el análisis y distorsiona la obra del poeta. Entiendo que el compromiso primero debe darse con la obra misma. Sólo a partir de ella deberá intentarse la aplicación de las teorías que sean afines a su propuesta literaria.

\section{Literatura, vida, pureza}

En el presente ensayo propongo un nuevo ahondamiento en la figura y en la poesía de Oliverio Girondo y una revalorización de su obra más densa y menos difundida: En la masmédula (1956). Con anterioridad publicó Veinte poemas para ser leidos en el tranvia (1922), Calcomanias (1925), Espantapájaros (1932), Interlunio (1937) y Persuasión de los días (1942). El poeta argentino representa la cifra de la coherencia entre literatura y vida; paralelamente a esta relación subyace la idea de una pureza rectora de su poesía. ${ }^{13}$

Dicha pureza difiere del llamado purismo, corriente poscubista cuyos teóricos fueron Amédée Ozenfont y Le Corbusier. Con el término "pureza", aplicado a nuestro poeta, tampoco hago referencia al "arte puro", denominación con que Valéry continuó el simbolismo francés y que tuvo repercusión en el grupo mexicano de Los Contemporáneos, como ha observado Anthony Stanton. ${ }^{14}$ Una primera caracterización de la obra de Girondo desde este punto de vista es la que proporciona Enrique Molina, quien se refie-

${ }^{13}$ El concepto de "pureza" ha sido tratado sobre todo en dos campos de la investigación: historia de las religiones y filosofia. La propia reinterpretación platónica de la idea de "pureza" corresponde precisamente al momento de escisión entre la pureza desde el punto de vista religioso - concepto que se encuentra en varias culturas-y la pureza como vía de conocimiento. En lo que sigue expongo algunos aspectos de la acepción griega. Gloso a Paul Poupard (Diccionario de las religiones). En la base de esta concepción se encuentra, como sugiere la semántica, la mancha culpabilizadora y el temor a los dioses que ésta ocasiona. En este aparato conceptual subyace la idea de la dualidad del hombre y la creencia de que el alma puede aislarse del cuerpo para recuperar su verdadera identidad. Las ideas de pureza existen en muchas culturas. Entre los griegos, uno de los primeros en tomarla fue Pitágoras. Según cuenta Platón (en el Fedón), Pitágoras instituyó un verdadero género de vida con prohibiciones materiales y propiciaba una ascesis que favorecía el recuerdo de existencias anteriores.

14 Anthony STANTON, "Los Contemporáneos y el debate en torno a la poesia pura", en Rafael Olea Franco y A. Stanton, eds., Los Contemporáneos en el laberinto de la critica. 
re específicamente al universo sonoro de Girondo como una "áccesis", 1 " esto es, como una paulatina pérdida del referente en favor de la ingravidez del lenguaje.

El concepto de "pureza" que permite vincular la vida y la obra de Girondo está más próximo al ámbito del pensamiento filosófico. ${ }^{16} \mathrm{En}$ la antigüedad fue Platón el que recogió las ideas existentes en torno a la pureza. Para él la purificación resume todo el proceso filosófico porque libera al alma del cuerpo. El alma tiene, pues, una función cognitiva. Mediante el alma se accede al mundo de las Ideas, afin a ella misma; es un mundo puro, sin mezcla, divino. ${ }^{17}$

La ascesis girondiana guarda relación con la noción whitmaniana de una "comunión plenaria" con el cosmos, que puede entenderse asimismo como la conciencia pitagórica de la armonía universal. Estas relaciones de Girondo con la pureza se pueden apreciar con mucha claridad en los poemas agrupados en Persuasión de los días (1942).

El concepto de ascesis o búsqueda de pureza, repito, funciona no en el sentido ético del término sino en el sentido filosófico de ausencia de mezcla, de simplicidad.

Los distintos periodos de la poesía de Girondo representan un progresivo alejamiento del referente hasta llegar a una ausencia total del mismo en En la masmédula, su obra final. Este alejamiento significa, en un registro filosófico, el inicio de una "desmezcla" que simboliza, como he señalado, una búsqueda de la pureza. Ésta se inicia con un asombro inicial ante la realidad, descrita desde los viajes por el mundo (como se puede apreciar en Veinte poemas para ser leidos en el tranvía y en Calcomanias). Como producto de esta observación Girondo pinta una realidad transfigurada por el grotesco, a causa del autoritarismo y el sexismo sociales. Así, la etapa primera es "impura". El proceso de "desmezcla" se puede apreciar en la etapa de transición. El candor y la pureza moldean el erotismo que paulatinamente se va desprendiendo del deseo: el amor sólo es posible con mujeres que vuelan. (En una sorprendente conversación que sostuve en 1996 con el escritor argentino Juan Filloy, éste admitió, a sus ciento dos años, haber sido un escritor profundamente erótico pero poco sensual. Es posible plantear, pues, la separación de la sensualidad o el deseo del erotismo. Correlativa-

15 "Hacia el fuego central o la poesía de Oliverio Girondo", estudio preliminar a las Obras completas de Oliverio Girondo. El término “áccesis" es un neologismo que emplea Molina. Prefiero sustituirlo por "ascesis".

${ }^{16} \mathrm{Cf}$. José Ferrater MORA, Diccionario de filosofia.

${ }^{17}$ Cf. P. POUPARD, op. cit. 
mente, la pureza, vinculada a la simplicidad, no necesariamente debe implicar el asombro, la ingenuidad y la inconciencia infantil, aunque la infancia constituye un importante punto de referencia.) En En la masmédula se supera el vuelo, terrestre al fin, para acceder (tal vez la "áccesis" de Enrique Molina sugiera esta idea) a un ámbito de ingravidez y oquedad: representa la cima del erotismo y la pureza, es decir, la resolución de términos que normalmente se configuran como antitéticos.

Según Vladimir Jankélévitch, ${ }^{18}$ las personas no pueden afirmar de si mismas la calidad de "puras". El adjetivo "puro" jamás será el atributo de una afirmación categórica de la primera persona del singular. Ningún hombre puede afirmar un juicio de valor de tal naturaleza. No es el sujeto que habla el que juzga. Sin embargo, añade: "l'enfant est l'innocence mème, ou le pureté substantielle, mais par définition, il n'en sait rien; l'enfant est pur, mais il ne le sait pas, et il n'est précisément pur qu'á la condition de l'ignorer; l'adulte conscient le saurait et mème ne le saurait que trop s'il l'était, mais justement parce qu'il le sait il ne l'est plus!" (p. 6).

Así, debemos reconocer que la impureza, en el sentido de mezcla, es palpable en la contingencia de nuestra vida cotidiana (esa manifestación "admirable y modesta de lo absurdo", nos dice Girondo, ya desde 1922). La pureza, en sí misma, no existe. La pureza absoluta y superlativa es aquella que no puede profesarse sin contradecirse; es la blancura y la transparencia absolutamente diáfanas. El que aprecie el valor de su propia pureza es, ipso facto, impuro. Como decía Angelus Silesius: "Lo que soy lo ignoro y no soy lo que se". ${ }^{19} \mathrm{El}$ ser y el saber, según este retruécano, se excluyen porque siempre hay en el ser humano un sustrato que permanece desconocido.

De acuerdo con lo anterior, Girondo no es un poeta metafísico ni es un poeta del conocimiento. No es, en otras palabras, un poeta del decir. Girondo es, en atención a la etimología de poiesis (hacer), el "hacedor" de lenguajes que trastocan la sintaxis, el "hacedor" que forja con ella la expresión de un sentido inédito en la poesía argentina contemporánea: una poesía alejada de la referencialidad y que ha logrado, como alguna vez lo quiso Breton, la superación de los contrarios como la máxima aspiración, en su caso, del surrealismo. Los contrarios que Girondo aúna maravillosamente son el erotismo y la pureza. La fuerza experimentadora ha surgido de la prodigiosa capacidad de Girondo para enfrentarse al lenguaje. Y el lenguaje de Girondo es la expresión del sentido de un retorno al estado primigenio de la inocencia, sin él saberlo, que le ha permitido, como a los niños, jugar con la mez-

18 Vladimir JANKÉLÉVITCH, Le pur et l'impur.

${ }^{19}$ Citado por V. JankÉLÉVITCH, ibid., p. 6. 
cla: "la pura impura mezcla que me merma los machimbres / el almamasa tensa las tercas hembras tuercas", leemos en el poema inicial de En la masmédula. La mezcla es la impureza, acaso la misma de ese mundo que recorrió en su juventud, la que el "hacedor" volvió pura, a pura fuerza de sonidos, aprovechados en los sentidos de Mallarmé y de Verlaine. Si, como decía Borges al evocar al Platón del Cratilo, "en las letras de rosa está la rosa", del mismo modo en el sonido de "lubidulia" debe estar, en efecto, "lubidulia", una realidad ingrávida que navegará para siempre en el oído de quien lo escuche:

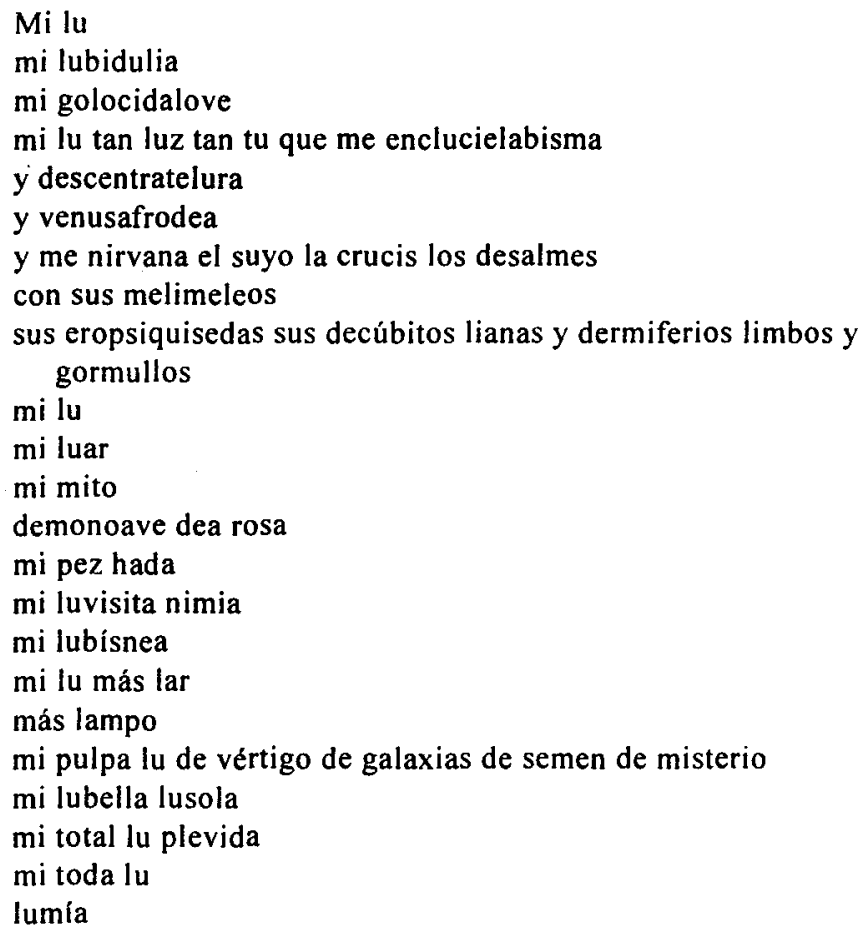

Las connotaciones semánticas de las palabras "condensadas" no necesitan muchas explicaciones. La significación "luz", el objeto del deseo, se identifica con la pasión amorosa. La luz, fuerza que alimenta y da vida, al mismo tiempo cura el dolor. Son tópicos de la literatura de todos los tiempos. Pero se trata de un amor sublimado puesto que entre "luz" y "fuego" hay distancia. El fuego es esa parte de la llama doble que significa la pasión carnal. En el poema existe la otra parte de la llama, el fuego del lar que es, 
como se sabe, doméstico, apacible. Así pues, la luz del poema es la de un amor sublimado. Se trata, entonces, de un erotismo "casto y espontáneo", el mismo que nuestro autor vio en Chagall y que tal vez lo inspiró para la creación de esa mujer etérea que aparece en Espantapájaros.

\title{
Un vuelo sin orillas
}

Consideremos al poeta en el espacio argentino. Buenos Aires, en los años veintes y treintas, se literaturiza. Se hace posible, si no vivir de la literatura, cuando menos vivir en la literatura, como lo testimonian las vidas de Leopoldo Lugones, Ricardo Güiraldes, Victoria Ocampo, Macedonio Fernández y el mismo Oliverio Girondo, entre otros. Son tiempos en los que se aprovechan al máximo las políticas educativas iniciadas a finales del siglo XIX: los suplementos literarios de La Nación y La Prensa ya han adquirido renombre, la revista Sur, a partir de 1931, se convierte en un símbolo de la aproximación cultural del Buenos Aires cosmopolita y el tempo europeo, proliferan casas editoras y se reactiva la Biblioteca Nacional. En suma, Buenos Aires se "sincroniza" con Europa y en su cosmopolitismo deja atrás la Gran Aldea. Éste es el contexto de la famosa "querella" entre "bandos" literarios: grupo de Boedo y grupo de Florida (éste con su órgano principal, el periódico Martin Fierro, donde se perfilan liderazgos como los de Jorge Luis Borges, Ricardo Guiraldes, Macedonio Fernández y el mismo Oliverio Girondo, todos de diferente estilo).

La evolución de Girondo desde 1922 hasta 1956 significa un progresivo paso de lo referencial a lo no referencial, del mundo al "vuelo sin orillas". En el primer caso, lo exterior se transfigura por el uso peculiar de la metáfora. Ésta va mucho más allá de una simple sustitución ornamental. El referente adquiere una nueva forma, interpretado desde diversos enfoques. “iEs tan real el paisaje que parece fingido!", escribe al referirse a Andalucía en 1925. Pero en su última obra Girondo indaga en las profundidades del ser. El viaje interior desplaza el interés por el mundo. El yo se autonombra inquisitivamente:

\author{
Eh vos \\ tatacombo \\ soy yo \\ di \\ no me oyes \\ tataconco \\ soy yo $\sin$ vos
}


sin voz

aquí yollando

con mi yo sólo solo que yolla y yolla y yolla (p. 435)

Como dijimos, en la primera época predominan las metáforas verbales. En las siguientes etapas ya no existe ese Girondo fuertemente armado de recursos ultraístas y es posible advertir el desplazamiento de la metáfora en favor de otras estrategias no ultraistas..$^{20} \mathrm{La}$ explicación radica en que la metáfora ha servido para nombrar y deformar el referente, es decir, ha sido un instrumento para la representación. Pero estos cambios dejan intacto al Girondo arriesgado y contundente, al Girondo pleno de sorpresas y audacias. Hay un empleo creativo de otras formas que posibilita el paulatino alejamiento del referente. Dentro de las llamadas formas hay que destacar un cambio en la presentación de las primeras dos obras, que son libros de viaje en el sentido convencional. En Espantapájaros, los veinticuatro textos numerados y anunciados por un caligrama sugieren la continuidad del viajero, que ahora se arriesga consigo mismo. Viaje interior, en suma, propicia el inicio del proceso en que la mezcla empieza a descomponer sus elementos. En la etapa final, especialmente en En la masmédula, Girondo experimenta con el ritmo y la sonoridad. El sentido nace de la forma, de los juegos silábicos y fonéticos. La constancia de la pureza sigue manifiesta, pero también se afirma el erotismo como elemento que, si en muchas ocasiones se contrapone a la ascesis, en otras, paradójicamente, se corresponde con ella.

$\mathrm{El}$ análisis de ciertos poemas eróticos (como el "Exvoto" a las chicas de Flores, el texto sobre la mujer etérea y "Mi lumía", arriba transcrito) conduce a la verificación de su búsqueda en este sentido. El análisis del lenguaje así lo confirma: el término pureza se relaciona, en efecto, con el erotismo y con la soledad.21

${ }^{20}$ Empleo el término "ultraismo" algunas veces como permutable con el término "cubismo" y otras veces con el compuesto "cubo-futurismo". Las denominaciones "europeas" son funcionales para describir la idea de descomposición, desconstrucción y descoyuntamiento de la realidad, asi como para marcar la caracteristica de la simultaneidad, visibles tanto en la pintura como en la poesía de la época. La corriente acuñada como "ultraísmo" especifica el fenómeno argentino y responde a los postulados que Borges resumió en 1921, a su regreso a la Argentina. Básicamente, se refiere al uso de la metáfora.

La forma indistinta en que a veces empleo los términos responde a las ideas que Jorge Schwartz ha expuesto en sus trabajos sobre la vanguardia hispanoamericana y en particular en la investigación comparativa entre Oliverio Girondo y Oswald de Andrade.

${ }^{21} \mathrm{Cf}$. Georges BatallLe, El erotismo; Severo SARDUY, "El barroco y el neobarroco", en César Fernández MORENo, comp., América Latina en su literatura. 
Podemos postular que Enrique Molina es el heredero de Girondo. Para sostener esto quizá sea adecuado retomar las aseveraciones iniciales, en las cuales sostengo que en Girondo hay una correspondencia entre literatura y vida. Esto no implica de ningún modo una valoración moralizante de la poesía, sino la posibilidad de tomar en cuenta uno de los caminos, en mi opinión, más productivos para abordar la poesía contemporánea. No se trata tampoco de atender a la simple tematización de la vida del poeta, sino de la inclusión de ésta como tarea poética. Con antecedentes en Wordsworth y el romanticismo, pero sobre todo desde Whitman, la relación vida-poesía ocupa un lugar fundamental en la creación contemporánea. Si, como escribe Tomás Segovia, el público del poeta es al mismo tiempo tan cercano y concreto como el de la intimidad familiar y tan abstracto y universal como la humanidad toda, ${ }^{22}$ se comprende que el destinatario de la escritura oscile entre la máxima abstracción, siempre ideal, y la tarea de escribir para un lector entendido como un "tú" que no puede prescindir de las notas existenciales que incluyen necesariamente al "nosotros" y al "yo" y que remite, una vez más, a la vida del poeta.

Noé Jitrik sostiene en una entrevista que me concedió en 1996 que el uso de categorías como literatura y vida puede conllevar el peligro de una reducción de la poesia a la ética. Afirma el crítico argentino que el sistema semiótico se dispara y se proyecta mucho más allá del autor. No obstante, mantengo la convicción de que en Girondo hay, en efecto, dicha correspondencia, lo cual no significa que yo la postule como una ley que debe comprobarse en todos los autores. Simplemente, en el caso de Girondo, me pareció una muestra de autenticidad y coherencia. Así, la afirmación de que Enrique Molina (1910-1996) es el heredero de Girondo debe tomarse con cuidado. Es el heredero -en palabras de Jitrik - como tributo a una amistad. En términos poéticos Molina fue un surrealista más ortodoxo. La herencia de Girondo, en un sentido más literario, es más clara en la obra de Leónidas Lamborghini: tiene que ver, en este caso, con una violencia en el orden de la escritura y no en el orden de la representación. Es una experiencia valiosa porque simboliza una apropiación del lenguaje por una vía que a Lamborghini le ha resultado eficaz, la parodia:

${ }^{22} \mathrm{Cf}$. los excelentes ensayos titulados "El infierno de la literatura" (pp. 195-217), "El poeta y el público" (pp. 331-348) y "El sexo del arte" (pp. 413-430), en Ensayos (Actitudes/Contracorrientes). De las reflexiones de Segovia se puede inferir que él estaría de acuerdo en que la relación literatura-vida, sobre todo en el caso de los poetas, es una postulación válida para el análisis. 


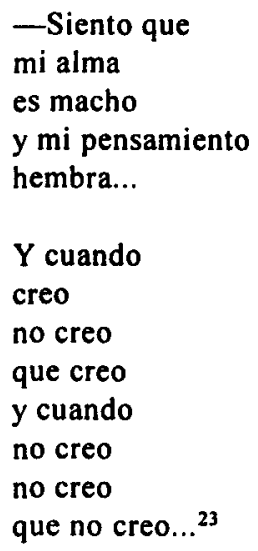

\section{Conclusiones}

Girondo indaga sobre la realidad en busca de la pureza, diluida en el mundo contemporáneo. La pureza es una exigencia de absoluto. Desde este punto de vista Girondo ha sido un poeta extremista, productor de una poesía extremada, en el sentido que Maravall le ha dado a este término en relación con el barroco. ${ }^{24}$ Era extremista y extremado no sólo en poesia. Lo era también en su postura ante la vida: desechó todos los convencionalismos culturales de su época, se aisló del mundillo literario, se acercó a escritores más jóvenes.

El término "pureza" connota indebidamente una búsqueda de lo natural. Por ello es necesario eliminar las resonancias morales al estilo de Rousseau. En todo caso, la diferencia con el filósofo francés es el asombro por la vida, más cercano al fervor whitmaniano. Así pues, en la ascesis girondiana si bien no necesariamente se encuentra una relación entre literatura y vida, hay ciertos elementos de alguna correspondencia, como la vuelta a la infancia, la búsqueda de la pureza y la exigencia de absoluto.

\section{Bibliografía}

BATAILLE, Georges, El erotismo. Barcelona, Tusquets, 1992.

\footnotetext{
${ }^{23}$ Leónidas LAMBORGHINI, Odiseo confinado, p. 144.

24 José Antonio Maravall, La cultura del barroco.
} 
CORRo, Gaspar Pío del, Oliverio Girondo. Los límites del signo. Buenos Aires, Fernando Garcia Cambeiro, 1976.

FERRATER MORA, José, Diccionario de filosofia. Barcelona, Ariel, 1994.

Gola, Patricia, Vanguardismo y ruptura en la poesia de Oliverio Girondo. México, UNAM, 1986. Tesis de licenciatura. Inédita.

GRÜNFELD, Mihai, Oliverio Girondo y la poesia de viaje. Universidad de California, 1987. Tesis doctoral.

"Hacia el fuego central o la poesía de Oliverio Girondo", estudio preliminar a las Obras completas de Oliverio Girondo.

JANKÉLÉVITCH, Vladimir, Le pur et l'impur. Paris, Flammarion, 1960.

JUZYN-AMESTOY, Olga, Oliverio Girondo: la realidad de la palabra. Universidad Brown, 1990. Tesis doctoral.

LAMBORGHINI, Leónidas, Odiseo confinado. Buenos Aires, Van Riel, 1992.

Maldoror. Montevideo, 16 de noviembre de 1982, pp. 22-35.

MARAVall, José Antonio, La cultura del barroco. Barcelona, Ariel, 1980.

MORAnd, Paul, Papiers d'identité. París, Grasset, 1931.

NELSON, Daniel E., Five central figures in Argentine avant-garde art and literature: Emilio Pettoruti, Xul Solar, Oliverio Girondo, Jorge Luis Borges, Norah Borges. Universidad de Texas, 1989. Tesis doctoral.

NÓBILE, Beatriz de, El acto experimental. Oliverio Girondo y las tensiones del lenguaje. Buenos Aires, Losada, 1972.

"Pintura Moderna". (Colección de Rafael A. Crespo.) Prólogo de Oliverio Girondo. Buenos Aires, Imprenta Colombo, Museo Nacional de Bellas Artes, 1936, 325 pp. ilus. (Exposición en el Museo Nacional de Bellas Artes, Buenos Aires, 14 de agosto al 6 de septiembre de 1936.) Reproducido en Obras completas. Buenos Aires, Losada, 1968, p. 218. 
POUPARD, Paul, Diccionario de las religiones. Barcelona, Herder, 1987.

SARDUY, Severo, "El barroco y el neobarroco", en América Latina en su literatura, César FERNÁNDEZ MORENO, comp. México, Siglo XXI, 1978.

SCHWARTZ, Jorge, "A trajetória maismedular de Oliverio Girondo", en Oliverio Girondo. A pupila do zero. San Paulo, Iluminuras, 1995.

SCHWARTZ, Jorge, Vanguardia y cosmopolitismo en la década del veinte: Oliverio Girondo y Oswald de Andrade. Universidad de San Paulo, 1979. Tesis doctoral publicada posteriormente con el mismo título. San Paulo, Perspectiva, 1983.

SEGOVIA, Tomás, "El infierno de la literatura" (pp. 195-217), "El poeta y el público" (pp. 331-348) y "El sexo del arte" (pp. 413-430), en Ensayos (Actitudes/Contracorrientes). México, UAM, 1988.

STANTON, Anthony, "Los Contemporáneos y el debate en torno a la poesía pura", en Rafael OLEA FRANCO y Anthony STANTON, eds., Los Contemporáneos en el laberinto de la crítica. México, El Colegio de México, 1994. 